\title{
Old but gold: the role of drug combinations in improving response to immune check-point inhibitors in thoracic malignancies beyond NSCLC
}

Luca Cantini ${ }^{1} \oplus$, Federica Pecci ${ }^{1}$, Filippo Merloni ${ }^{1}$, Andrea Lanese ${ }^{1}$, Edoardo Lenci $^{1}$, Francesco Paoloni ${ }^{1}$, Joachim G.J.V. Aerts ${ }^{2,3}$, Rossana Berardi ${ }^{*}{ }^{*}$

${ }^{1}$ Clinical Oncology, Università Politecnica delle Marche, A.O.U. Ospedali Riuniti, 60126 Ancona, Italy

${ }^{2}$ Department of Pulmonary Medicine, Erasmus MC, 3015 CE Rotterdam, The Netherlands

${ }^{3}$ Erasmus MC Cancer Institute, Erasmus MC, 3015 CE Rotterdam, The Netherlands

*Correspondence: Rossana Berardi, Clinical Oncology, Università Politecnica delle Marche, A.O.U. Ospedali Riuniti, Via Conca 71,60126 Ancona, Italy. rossana.berardi.it@gmail.com; r.berardi@univpm.it

Academic Editor: Nicola Normanno, Istituto Nazionale Tumori-IRCCS-Fondazione G. Pascale, Italy

Received: November 5, 2020 Accepted: December 8, 2020 Published: February 28, 2021

Cite this article: Cantini L, Pecci F, Merloni F, Lanese A, Lenci E, Paoloni F, et al. Old but gold: the role of drug combinations in improving response to immune check-point inhibitors in thoracic malignancies beyond NSCLC. Explor Target Antitumor Ther. 2021;2:1-25. https://doi.org/10.37349/etat.2021.00030

\section{Abstract}

The introduction of immune checkpoint inhibitors (ICIs) in non-oncogene addicted non-small cell lung cancer (NSCLC) has revolutionized the treatment scenario and led to a meaningful improvement in patient prognosis. Disappointingly, the success of ICI therapy in NSCLC has not been fully replicated in other thoracic malignancies as small cell lung cancer (SCLC), malignant pleural mesothelioma (MPM), and thymic epithelial tumors (TETs), due to the peculiar biological features of these disease and to the difficulties in the conduction of well-designed, biomarker-driven clinical trials. Therefore, combination strategies of ICIs plus conventional therapies (either chemotherapy, alternative ICIs or targeted agents) have been implemented. Although first approvals of ICI therapy have been recently granted in SCLC and MPM (in combination with chemotherapy and different ICIs), results remain somewhat modest and limited to a small proportion of patients. This work reviews the trial results of ICI therapy in mesothelioma, SCLC, and TETs and discusses the potential of combining ICIs with old drugs.

\section{Keywords}

Immune checkpoint inhibitors, small cell lung cancer, mesothelioma, thymic epithelial tumor, immunooncology, immune modulating

\section{Introduction}

In the last decade, the treatment landscape of non-small cell lung cancer (NSCLC) changed dramatically, due to the introduction of targeted therapies and immunotherapy. The success of immune checkpoint inhibitor (ICI) therapy in non-oncogene addicted NSCLC [1-4] has led to investigation of these drugs into other thoracic 
malignancies such as small cell lung cancer (SCLC), malignant pleural mesothelioma (MPM), and thymic epithelial tumors (TETs).

Clinical trials testing immunotherapy in patients with these thoracic cancers have been conducted or are ongoing [5-7]. Unlike NSCLC, where ICIs have represented a breakdown in the treatment armamentarium and have received approval in different settings of disease [8-10], the improvement in outcomes with ICI therapy in rarer thoracic tumors has been somewhat modest and limited to a small proportion of patients [11].

The peculiar pathogenesis underneath these tumors, along with multiple biases in the design of clinical trials were most probably responsible for delaying the availability of effective immunotherapies. Similar to NSCLC, there might be a subgroup of patients more likely to benefit from ICIs [12], but relevant biomarkers have not been determined yet. In addition, lack of funding for clinical research in rare cancer entities such as MPM and TETs has probably precluded the conduction of robust clinical trials that could address specific research questions [13].

What emerges from these recent approvals and from the disappointing results of single-agent ICIs, is the need to properly combine ICIs with other drugs in order to overcome resistance, improve clinical activity and better handle side effects of ICIs in both SCLC, MPM and TETs.

Other conventional therapies such as chemotherapy and targeted therapy, even the ones with different oncological indications, or more recently developed immunotherapies might synergize with ICIs to counteract the immunosuppressive environment of these tumors (Figure 1).

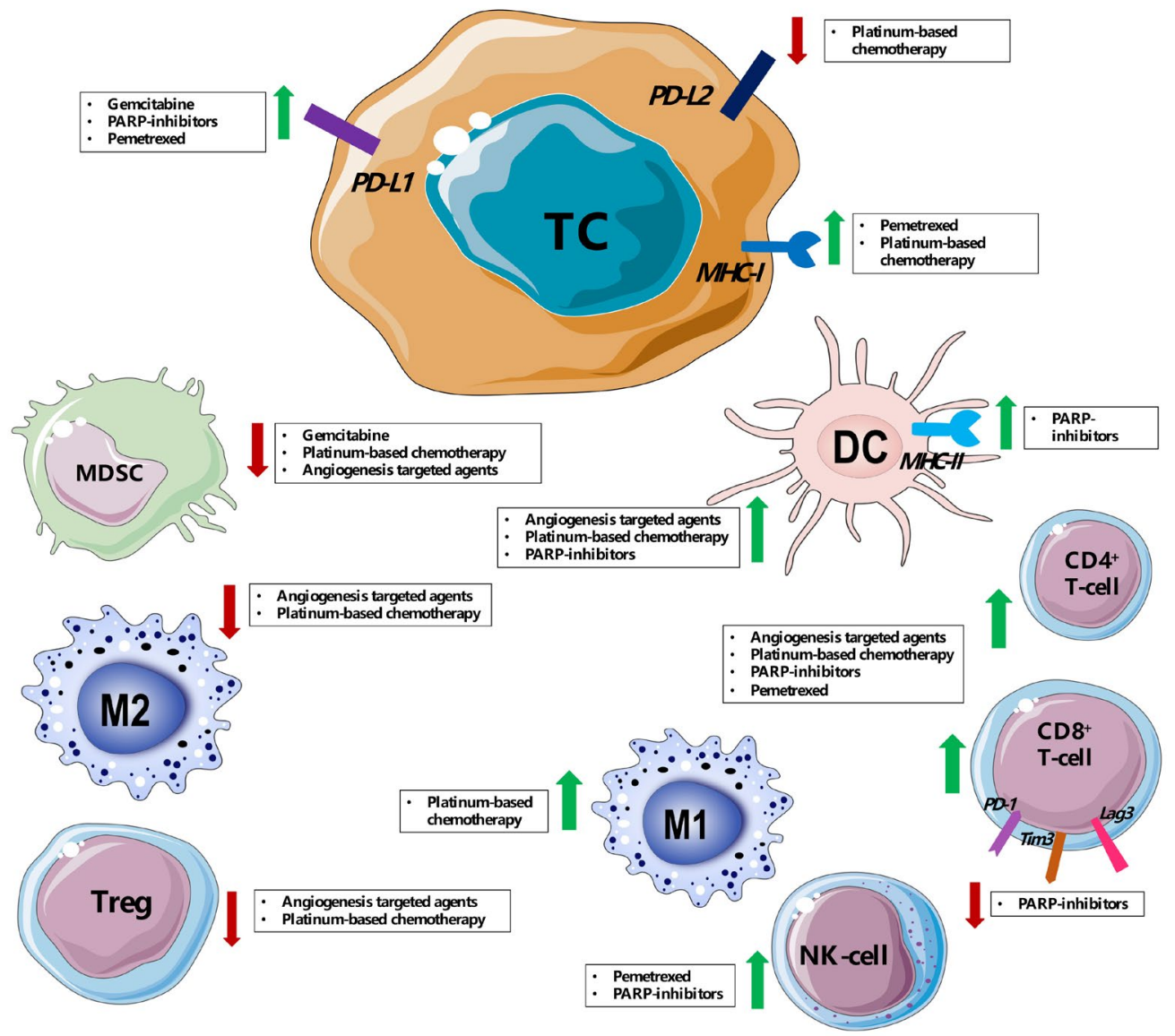

Figure 1. Immune modulation of tumor microenvironment mediated by main drugs [platinum-based chemotherapy, pemetrexed, angiogenesis targeted agents (such as bevacizumab and sunitinib), poly (ADP-ribose) polymerase (PARP) inhibitors] used in combination with ICls in pre-clinical works and clinical trials for SCLC, MPM and thymic cancer. PD-L2: programmed death-ligand 2; DC: dendritic cell; TC: tumor cell; MHC-I: major histocompatibility complex class I; MHC-II: major histocompatibility complex class II; NK-cell: natural killer cell; MDSC: myeloid-derived suppressor cell; Tregs: regulatory T cells

In this review, we discuss the current role of ICI treatment in SCLC, MPM and TETs, by examining the results coming from already published clinical trials and explore future perspectives for novel combination therapies that could improve ICIs efficacy (Tables 1-3), by discussing their biological rationale as well as the importance of properly selecting patients for every treatment. 


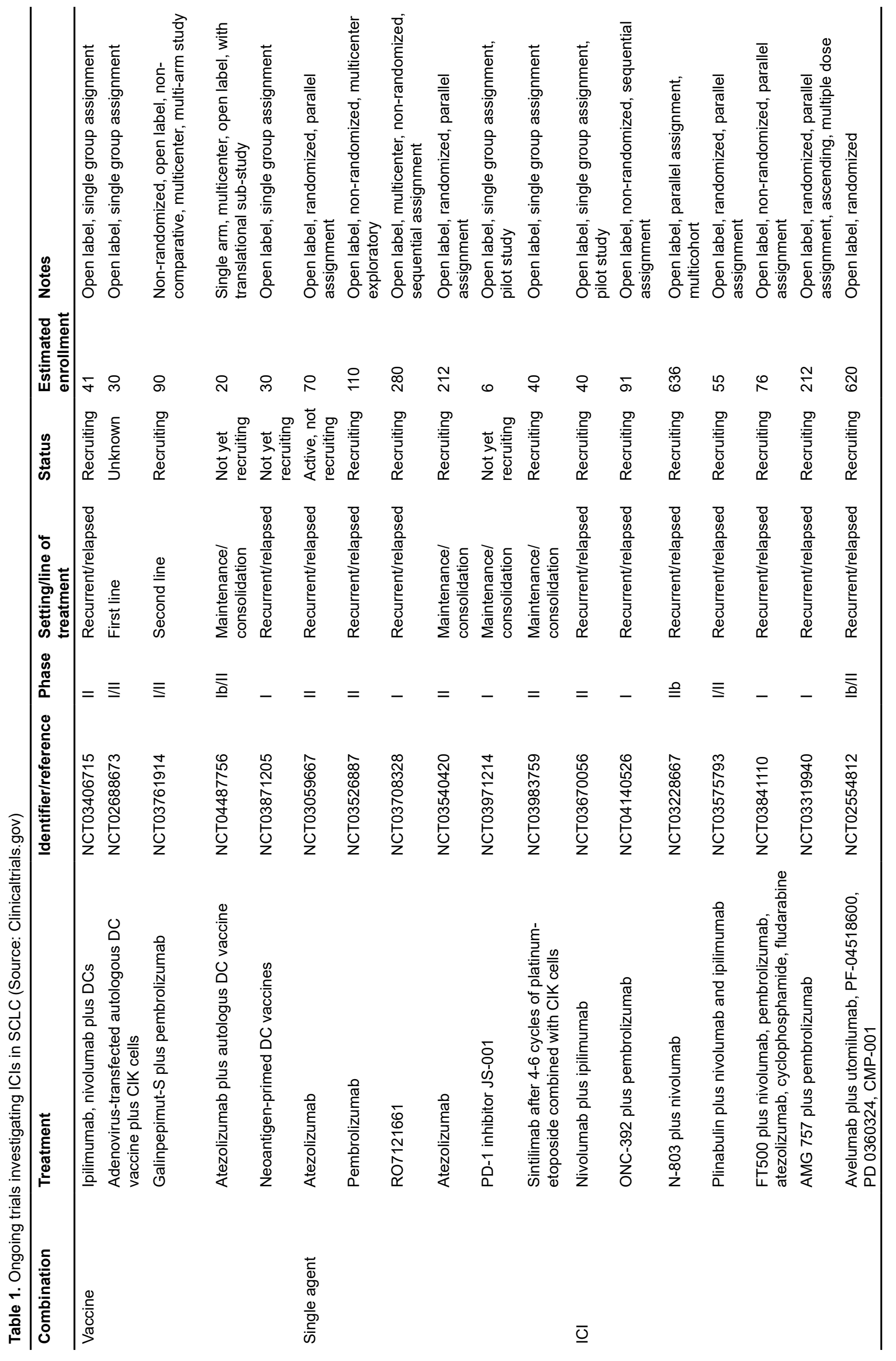




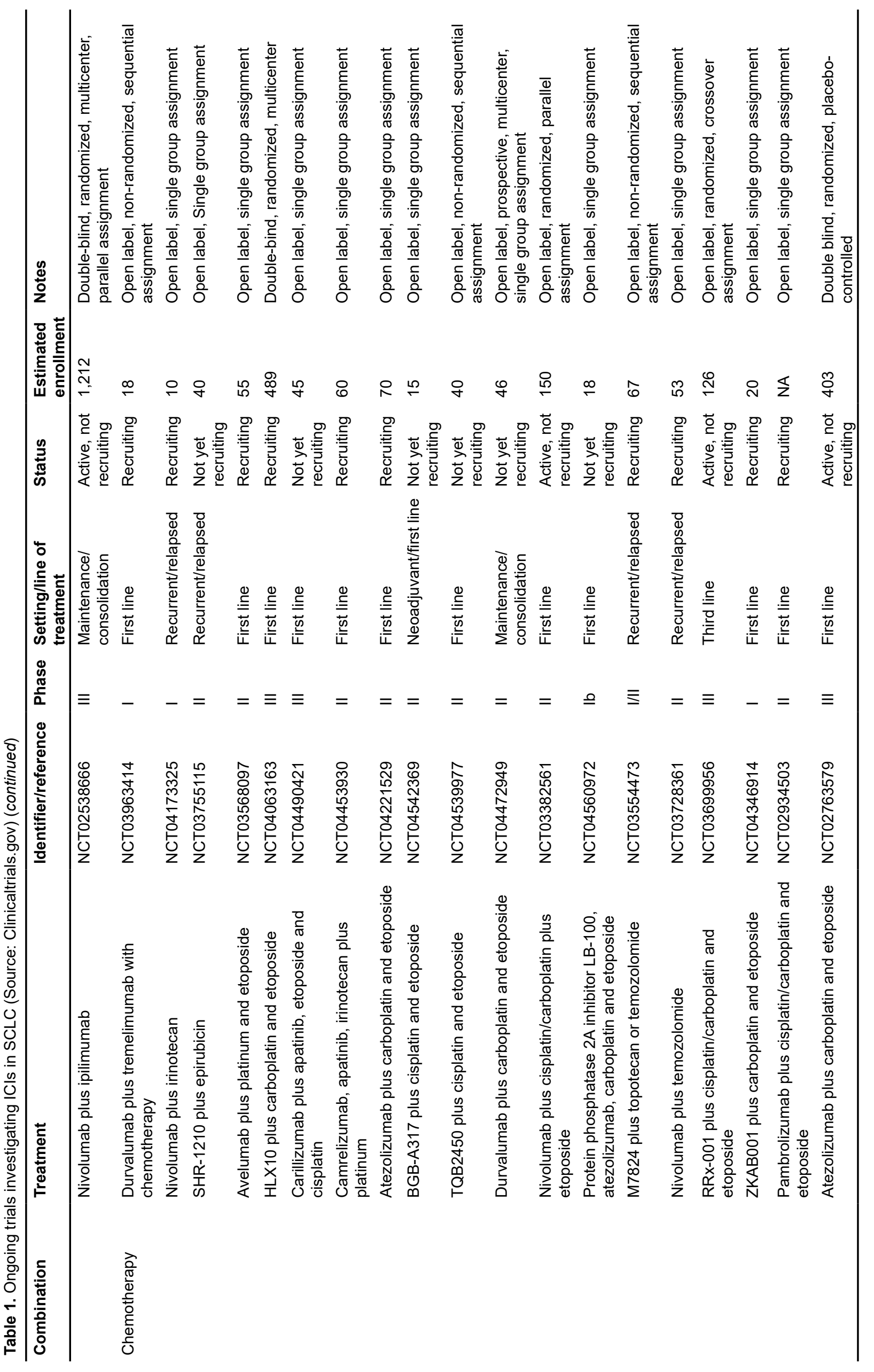




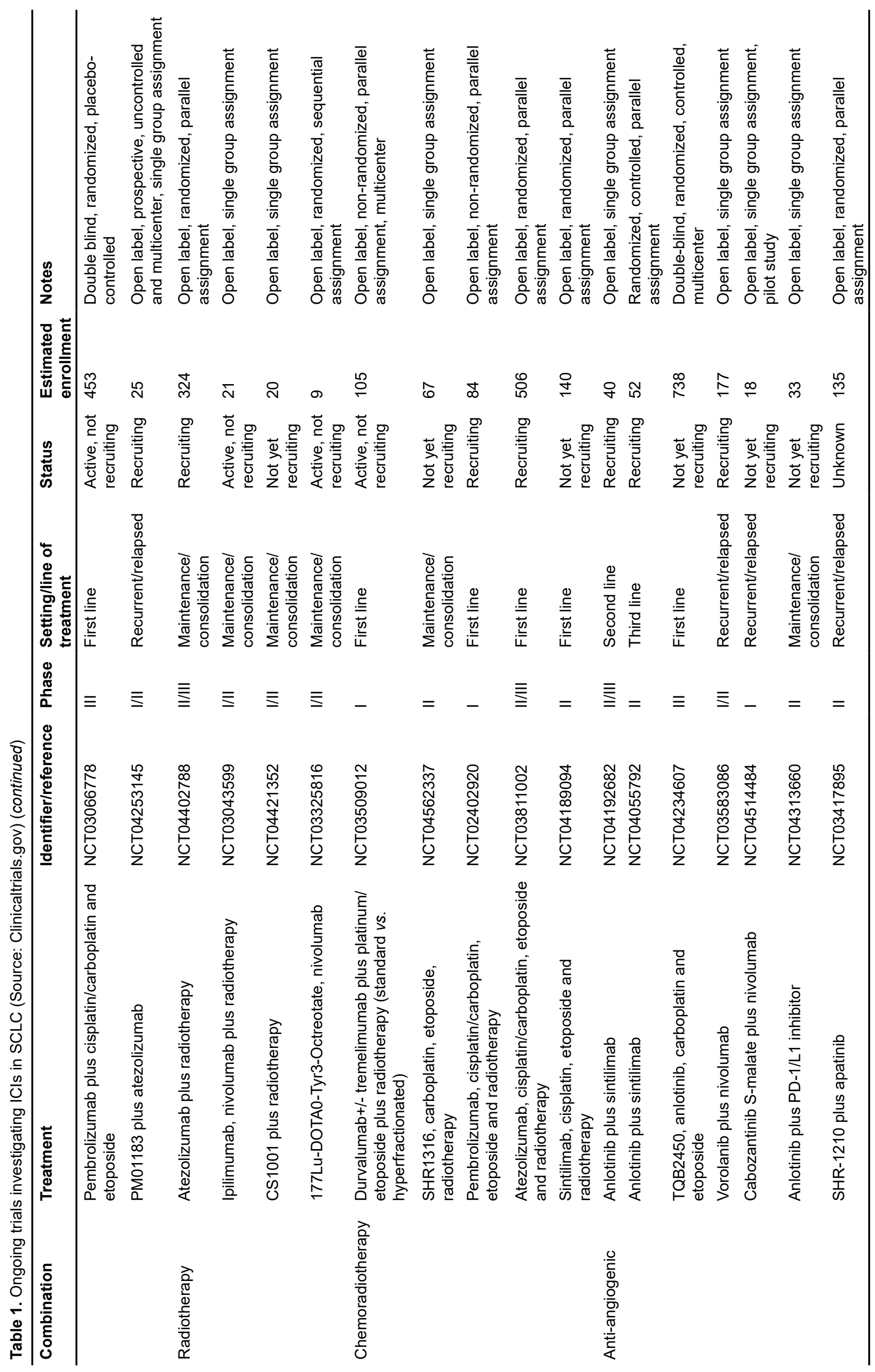




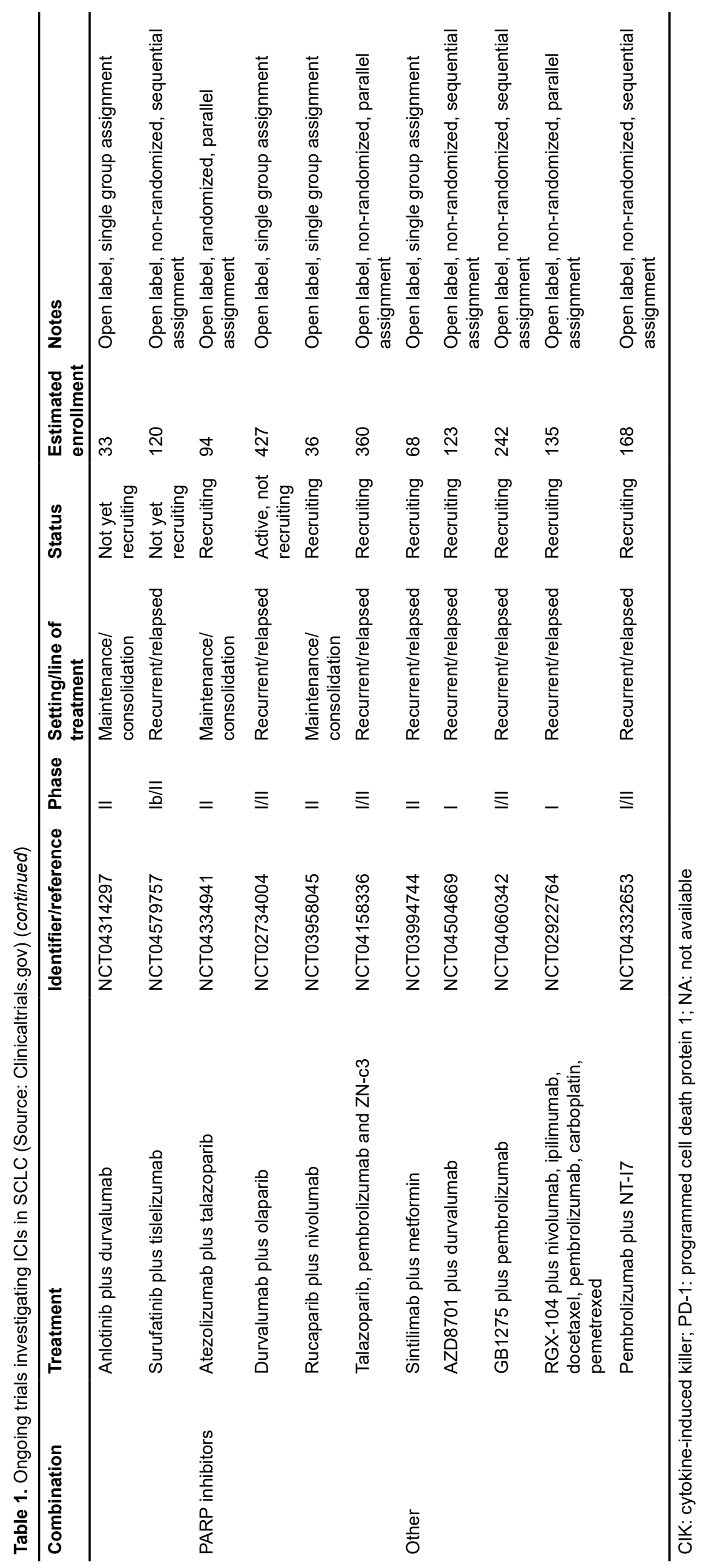




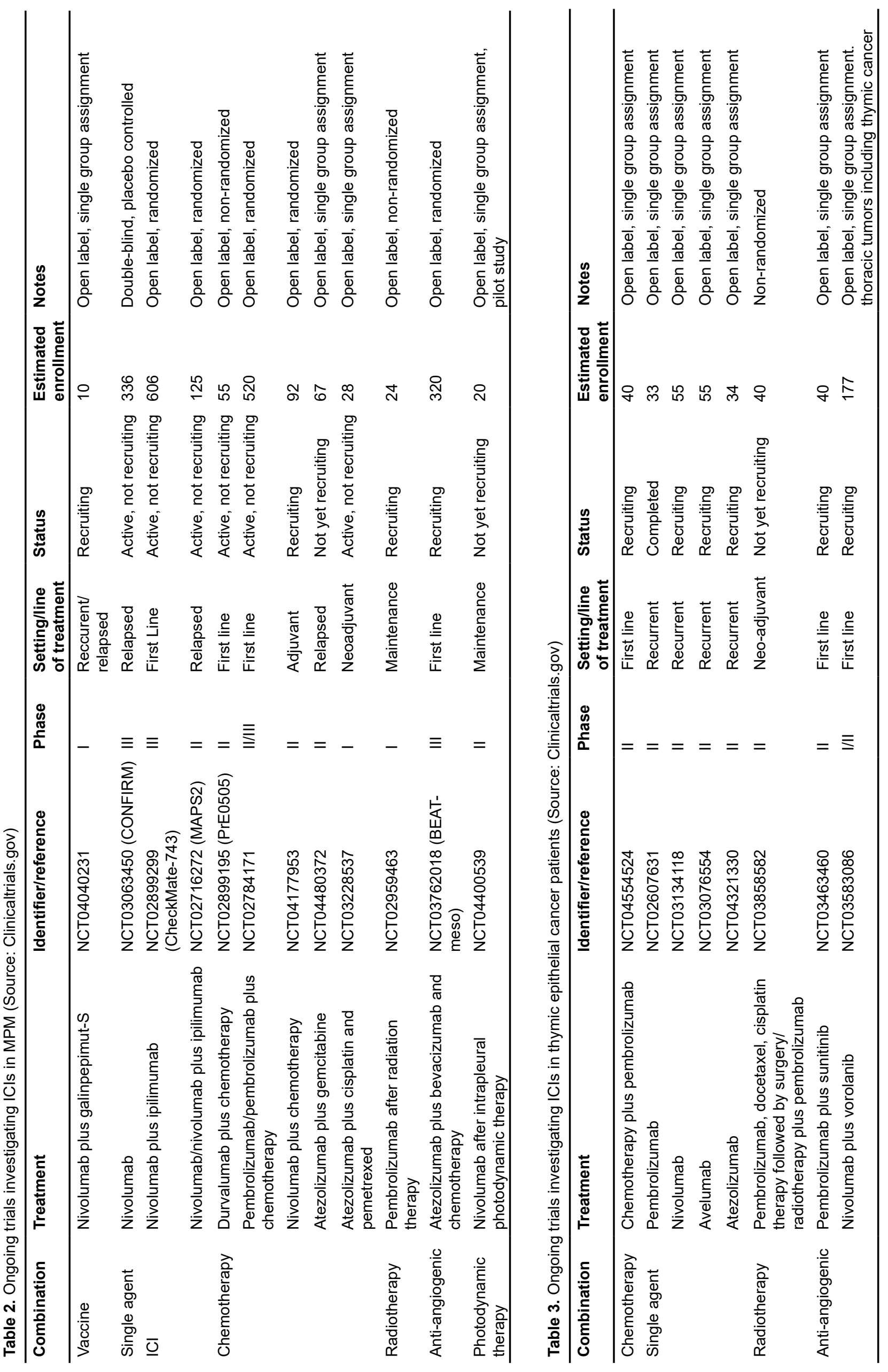




\section{SCLC}

Around 15\% of lung cancers is represented by SCLC, a neuroendocrine tumor characterized by a high growth fraction and an early development of distant metastasis. SCLC can be staged using both the American Joint Committee on Cancer (AJCC) TNM and the historical Veterans Affairs (VA) classification system, which has only 2 stages: limited (disease confined to the ipsilateral hemithorax, which could be included in a radiation therapy filed) and extensive (anything beyond limited stage). The clinical implication of VA system made it extremely useful even today in clinical practice as well as research.

SCLC is strictly related to tobacco use. Smoking is known to damage DNA [14] and consequently determine a high quantity of somatic mutations, the so-called as tumor mutation burden (TMB), that is a welldocumented biomarker for immunotherapy $[15,16]$. This feature can lead to a release of tumor neoantigens capable to stimulate an anti-tumoral immune response, thus representing the rationale for immunotherapy activity in this disease [17].

Unfortunately, ICIs showed only moderate benefit when investigated in SCLC. Although the phase I/II CheckMate-032 trial assessing nivolumab with or without ipilimumab in relapsed SCLC documented a 1-year overall survival (OS) of $42 \%$ for nivolumab/ipilimumab combination and $30 \%$ for nivolumab alone [18], the phase III CheckMate-331 reported that nivolumab was not superior to topotecan in patients with relapsed or progressed SCLC after a platinum-based treatment in terms of OS, progression-free survival (PFS) and objective response rate (ORR) [19]. Notably, a late separation of the curves and a potential activity in the platinum-refractory setting suggests a possible long-term benefit for a subgroup of patients.

The global, double-blind, phase III study compared nivolumab plus ipilimumab or nivolumab alone vs. placebo as maintenance therapy in patients with extensive SCLC who did not progress to first line platinum chemotherapy. Both nivolumab plus ipilimumab and nivolumab alone did not improve OS compare to placebo but maintenance immunotherapy appeared to improve PFS, with rates of patients who were progressionfree at six months of $20 \%$ and $21 \%$ for nivolumab with or without ipilimumab, respectively, versus $10 \%$ for placebo [20].

The KEYNOTE-028 and the KEYNOTE-158 trials documented a prolonged durable response for SCLC patients after 2 or more lines [21]. However, these two were a phase Ib and a phase II studies, respectively, and a large randomized controlled trial with pembrolizumab in relapsed SCLC is missing.

Noteworthy, due to the paucity of therapeutic alternatives, nivolumab and pembrolizumab got the FDA approval for the third or later line treatment for SCLC, based on CheckMate-032 and KEYNOTE-028/ KEYNOTE-158 trial results. In CheckMate-032 PD-L1 staining seems to not be a predictor of response to nivolumab [22]. Exploratory analysis from the KEYNOTE-158 suggested a role of PD-L1 expression in patients with SCLC who may response to pembrolizumab [21]. Both in CheckMate-032 and in KEYNOTE-158 patients characterized by a high TMB seems to better response to nivolumab or pembrolizumab but further studies are needed to identify new biomarkers of response.

Different mechanisms have been proposed to justify the poor effectiveness of immunotherapy in this disease, such as low PD-L1 expression, downregulation of MHC molecules, immunosuppression induced by SCLC cells and autocrine and paracrine regulation [23, 24].

Therefore, further treatment strategies are required to overcome these mechanisms of ICI resistance, and drug combinations seem to be a promising approach.

\section{Chemotherapy + ICIs}

Historically, first-line treatment in SCLC has been represented by chemotherapy with platinum (cisplatin or carboplatin) plus etoposide, which showed a good ORR of about $65 \%$, but an OS far less satisfying, around 9-10 months [25].

In NSCLC, the combination of chemotherapy and immunotherapy has become the new standard of care in first-line, with exciting results [26, 27]. The rationale behind this synergism is the immunogenic cell death (ICD) induced by cytotoxic therapy and the concurrent appearance of specific damage-associated molecular 
patterns (DAMPs) on the surface of the apoptotic cells, able to trigger an anti-tumor immune response by the promotion of DC maturation and activation of cytotoxic T lymphocytes (CTLS). Meanwhile, chemotherapy is thought to reduce the immunosuppressive activity of tumor microenvironment, specially downregulating the MDSCs [28, 29].

Using chemotherapy is even clinically more necessary in SCLC, being a rapidly progressive disease, which requires a rapid tumor shrinkage, therefore a monotherapy with ICIs may be too ineffective. Until now, two studies were published showing efficacy of chemotherapy combined with ICIs and imposing a new standard of treatment in the first-line setting of ES-SCLC.

In the phase III trial IMpower133, atezolizumab was administered with carboplatin and etoposide in patients with ES-SCLC who were not previously treated [5]. The study documented a significant prolonged OS and PFS for patients who received immunotherapy compared to those treated with chemotherapy plus placebo [median OS (mOS) 12.3 months vs. 10.3 months, $P=0.007$ with HR of 0.70 and median PFS (mPFS) 5.2 months vs. 4.3 months, $P=0.02$ with HR of 0.77 ], leading to a gain of $13.5 \%$ of patients alive at 1 year. ORR did not differ between the two treatments.

The second phase III trial showing benefit in adding immunotherapy to standard treatment as firstline in patients with ES-SCLC was the CASPIAN trial [6]. The trial had also an arm in which an anti-cytotoxic T-lymphocyte-associated protein 4 (CTLA4) was added (durvalumab plus platinum-etoposide regimen, durvalumab plus tremelimumab plus chemotherapy and the chemotherapy control arm), but the planned interim analysis did not include the tremelimumab arm.

The results were in line with those of the IMpower133. In fact, adding durvalumab (an anti-PD-L1 agent) to standard chemotherapy showed to significantly improve OS compared to control arm, 13.0 months vs. 10.3 months $(P=0.0047$ with HR of 0.73 ) with $34 \% v s .28 \%$ patients alive at 18 months, respectively. Recently, the trial update confirmed the benefit in OS (12.9 months vs. 10.5 months, $P=0.0032$ with HR of 0.75 , after a median follow up of 25.1 months). However, the study's third arm with tremelimumab did not meet the prespecified threshold for statistical significance $(P \leq 0.0418)$. Indeed, the median OS for this combination was 10.4 months vs. 10.5 months for control arm $(P=0.0451$, HR 0.82$)$ and the 18-months OS rates was $32.0 \%$ in the durvalumab + chemotherapy, $30.7 \%$ in the tremelimumab + durvalumab + chemotherapy, and $24.8 \%$ in the control arm; at 24 months, those rates were $22.2 \%, 23.4 \%$, and $14.4 \%$, respectively. Therefore, the only combination available in first-line remains without the CTLA4 inhibitor.

Although similar, the two studies have few differences such as the number of cycles of platinum-etoposide in the control group ( 4 for IMpower133 and 6 for CASPIAN) that could have influenced the outcome and the possibility of investigator's choice of platinum, which was possible in CASPIAN but not in IMpower133 (where only carboplatin was allowed). Interestingly, the last update of CASPIAN reported that durvalumab was associated with prolonged OS regardless of which platinum was administered.

Furthermore, two more studies investigated the association of standard platinum-etoposide chemotherapy with pembrolizumab, the phase III KEYNOTE-604 trial, or with nivolumab, the phase II ECOGACRIN EA5161. In the KEYNOTE-604, at final analysis (median follow up 21.6 months), 9\% of patients in the pembrolizumab arm and $1 \%$ in the control arm were still on treatment. In the intention-to-treat (ITT) population, the combination prolonged OS, but did not meet the prespecified significance threshold of $P=$ $0.0128(P=0.0164$ with HR of 0.80 , median OS 10.8 months vs. 9.8 months with or without pembrolizumab respectively), thus making the results of this study not as robust as the others with different ICIs [30]. Differently, the association of platinum-etoposide chemotherapy with nivolumab improved both the median OS and the mPFS compared to chemotherapy in the ITT population (median OS 11.3 months vs. 8.5 months, $P=0.038$ with HR of 0.67 and mPFS 5.5 months $v s .4 .6$ months, $P=0.012$ with HR of 0.65 ), giving a potential ICI alternative to atezolizumab and durvalumab.

Besse et al. [31] at ESMO 2020 presented the results of REACTION study, a multicenter open-label randomized phase II trial. They randomized patients with extensive SCLC, unselected for PD-L1, and controlled brain metastases who detained an objective response after 2 cycles of chemotherapy with platinum and etoposide to receive pembrolizumab in combination with four additional cycles of platinum etoposide then 
pembrolizumab up to 35 cycles (experimental arm) vs. four additional platinum etoposide cycles (control arm). Even though pembrolizumab combined with platinum and etoposide did not improve PFS, data showed benefit in OS [mOS was 12.3 months for the experimental arm and 10.4 for control arm $(P=0.097$, one-sided)], suggesting a potential role of pembrolizumab in first line treatment to improve chemotherapy efficacy.

Reck et al. [32], investigated the combination of ipilimumab plus etoposide and platinum vs. placebo plus etoposide and platinum in a phase III randomized trial including patients affected by extensive-stage disease SCLC. The addiction of ipilimumab to chemotherapy did not show significant improvement both in OS and in the other secondary endpoints (PFS, ORR).

In addition to studying different ICIs, changing chemotherapy backbone is also a possible strategy that is worth to investigate. Anthracyclines, for example, represent a potential therapeutic strategy for SCLC treatment in combination with cyclophosphamide, adriamycin and vincristine (CAV regimen) [33]. It is well documented their activity both in first-line, before cisplatin/etoposide therapy showed to be superior [34], and in second-line, where no differences in terms of ORR (18.3\% vs. 24\%, $P=0.285)$, median time to progression (12.3 weeks vs. 13.3 weeks, $P=0.552)$ and mOS ( 24.7 weeks $v s .25 .0$ weeks, $P=0.795)$ with topotecan were found [35].

Moreover, it was demonstrated that anthracyclines, in particular doxorubicin, enhance an anticancerimmune activity by inducing calreticulin (CRT) exposure on dying cancer cell, which is a fundamental step for ICD mediated by DCs. Interestingly, the same mechanism was not documented in etoposide-treated mice [36]. Therefore, it is possible to hypothesize that immunotherapy with different chemotherapycombination, based on doxorubicin rather than etoposide for example, could lead to more promising results [37].

A novel drug recently designed as orphan drug to treat patients with relapsed SCLC is lurbinectedin, that induces apoptosis by inhibition of RNA polymerase II through blocking trans-activated transcription. Lurbinectedin has demonstrated to be active as a single agent, in a phase II basket trial, as well as in combination with doxorubicin in a phase Ib, especially in patients with chemotherapy-free interval of 90 or more days, showing an ORR of $53 \%$ and a PFS of 5.7 months [38, 39].

Based on these encouraging results, a prospective, open-label, uncontrolled and multicenter phase I/ II study of lurbinectedin in combination with pembrolizumab in patients with relapsed SCLC is ongoing (NCT04358237).

To conclude, although the clinical impact of the studies reported above might be not so impressive compared to those achieved in other malignancies, these results further confirm that combining chemotherapy with ICIs could be a promising treatment option, and different ICIs as well as different chemotherapies need to be evaluated to find the most efficient association.

\section{DNA damage response (DDR) inhibitors + ICIs}

Another combination which is stimulating the scientific community is immunotherapy with DDR inhibitors. It has been demonstrated in breast cancer and NSCLC that treatment with PARP inhibitors enhances expression of PD-L1 by increasing cytosolic DNA and consequently activating the cyclic GMP-AMP synthase (CGAS)/stimulator of interferon genes (STING) innate immune signaling. The entire process stimulates PD-L1 expression and T cell recruitment [40-42].

A similar pathway has been described for SCLC. A pre-clinical study observed in murine models an increase of PD-L1 expression and T-cytotoxic infiltration, decreased T-cell exhaustion and tumor shrinkage when either olaparib (a PARP inhibitor) or prexasertib (a checkpoint kinase 1 inhibitor) were associated to an anti-PD-L1 agent [43]. On the contrary, the MEDIOLA trial, a phase II basket trial studying the association of olaparib and durvalumab in different relapsed cancers, did not observe a clinical efficacy in SCLC unlike other tumors, such as breast or ovarian cancers [44]. Among 38 patients with ES-SCLC, only 4 had a tumor response, according to RECIST criteria, and 11 experienced disease control. In line with this result, a single arm, phase II study documented a partial or complete response in only 2 out 19 patients, who started at the 
same time durvalumab and olaparib (rather than adding durvalumab to the PARP inhibitor afterwards as in the MEDIOLA trial) [45].

Nevertheless, some considerations have to be done before considering this combination a failure. Firstly, platinum and PARP1 resistance are correlated, and because both trials were conducted in patients with platinum resistance/refractory disease, it could be reasonable to speculate that the combination may be more active before the resistance is developed. Secondly, germline BRCA1 or BRCA2-mutation (which are wellknown biomarker of efficacy for PARP inhibitor in other malignancies) are very rare in SCLC, thus probably explaining a minor activity of this combination. Patient selection should be rather based on the expression of schlafen family member 11 protein (SLFN11), which is more often documented in SCLC and also related to PARP1 sensitivity $[46,47]$. Thirdly, patients who experienced response in both studies reported above had an inflamed phenotype (i.e. CD8-positive T-cells in direct contact with tumor) or PD-L1-positive staining within tumor cells. Therefore, selecting patients by clinical outcome, biomarkers or immune phenotypes might allow this combination to be more effective and useful.

\section{Angiogenesis targeted agents + ICIs}

Pro-angiogenic factors impact the immune surveillance in different ways, by suppressing the function of several immune cells [48], decreasing the leukocyte-endothelial interactions and hampering the infiltration of immune effector cells into the tumor microenvironment [49]. It has been shown that maturation of DC precursors is suppressed by high vascular endothelial growth factor (VEGF) levels [50]. On the contrary, proliferation of immune suppressive cells such as Tregs cells and immature myeloid cells, is promoted by high VEGF levels [51]. The SCLC tumor tissue expresses high levels of VEGFA, VEGF receptors and PD-L1 [52, 53]. A pre-clinical work analyzed the efficacy of an anti-PD-L1 and anti-VEGF drug combination in a mouse model of SCLC, showing a synergic positive effect. The study showed how ICIs combined with anti-VEGF modified the tumor-infiltrating $\mathrm{T}$ cells. In fact, on mice not treated, $\mathrm{T}$ cells were not present on the pulmonary tissue around the tumor and on the tumor infiltration, whereas on anti-VEGF treated mice CD4 $4^{+} \mathrm{T}$ cells infiltrated tumor, with $\mathrm{CD}^{+}$at tumor margin. In addition, on mice treated with only anti-PD-L1, CD4 ${ }^{+}$accumulated in tumor margin without invading tumor tissue; only on mice treated with combination of anti-VEGF and anti-PD-L1 it was possible to see $\mathrm{CD} 4^{+} \mathrm{T}$ cells and $\mathrm{CD} 8^{+} \mathrm{T}$ cells infiltrate tumor tissue. The results strongly recommend a combination therapy of anti-VEGF agents and ICIs for the treatment of patients with SCLC [54].

During last years, receptor tyrosin kinases (RTKs) have become the target of several drugs used in clinical practice in NSCLC [55], but no survival advantages were obtained from clinical trials that investigated tyrosine kinase inhibitors (TKIs) in SCLC patients. Cabozantinib is a small-molecule kinase inhibitor, with activity toward MET, VEGF receptors 2 (VEGFR2) and other tyrosine kinases like RET, KIT, AXL, and FLT3 [56]. Due to the inhibitory effect of cabozantinib on the VEGFR2, it might promote the reprogramming of the immunosuppressive tumor microenvironment and synergize with ICIs [51]. On this basis, an ongoing phase II trial is investigating the combination of cabozantinib, nivolumab and ipilimumab in patients with poorly differentiated neuroendocrine tumors (NCT04079712). Another phase I/II dose escalation and dose expansion trial of combination of oral vorolanib, a VEGFR/PDGFR dual kinase inhibitor, and nivolumab is ongoing in patients with NSCLC, SCLC and thymic carcinomas (TC) (NCT03583086).

In conclusion, since the impact of ICI monotherapy on SCLC is limited, further combination strategies with drugs able to modulate the tumor immune microenvironment may become a stronger weapon to improve SCLC patient outcome [57].

\section{MPM}

MPM is a rare and aggressive tumor arising from mesothelial cells of the pleura most commonly caused by mineral fibers (such as asbestos and erionite) exposure [58]. 
Even though surgery and radiotherapy play a role in this pathology, the current established therapy is still systemic chemotherapy with cisplatin and pemetrexed on the basis of the phase III EMPACHIS trial which demonstrated a 3-months survival benefit compared to cisplatin alone (12.1 months vs. 9.3 months) [59, 60].

The limited benefit of first-line chemotherapy and the lack of an effective second-line strategy, with exception of the reintroduction of a pemetrexed-based chemotherapy in patients with durable response to front-line chemotherapy [60] prompt the detection of new therapeutic strategies.

ICIs have been an active field of research in MPM both for their successes in other malignancies and the important role which immune system exerts in the pathogenesis of MPM [61]. Some cases of spontaneous MPM regression likely related to an activation of the immune system have been reported [62] and a worse outcome has been related with high $\mathrm{CD}_{163^{+}}$tumor-associated macrophages and low $\mathrm{CD}^{+}$tumor infiltrating lymphocytes $[63,64]$. Furthermore PD-L1 was shown to be highly expressed in MPM cells and PD-L1 positivity has proven to be an independent risk factor for survival in MPM patients [65].

Therefore, various PD-1/PD-L1 inhibitors have been investigated in patients with disease progression after first-line chemotherapy. In a phase II trial pembrolizumab showed a disease control rate (DCR) of $47 \%$ with a PFS of 4.5 months [66] while, in two phase II trials investigating single agent nivolumab, the PFS was 2.6 and 6.1 months, respectively [67, 68]. Similar results were reported for avelumab [69].

A randomized study comparing pembrolizumab with chemotherapy (gemcitabine or vinorelbine) in MPM patients with disease recurrence reported almost equal PFS (2.5 vs. 3.4) and OS (10.7 vs. 11.7) between the two groups [70]. A phase III trial (CONFIRM trial) with randomization to nivolumab or placebo in MPM patients which progressed after at least 2 chemotherapy lines is currently ongoing [71].

Despite initial promising results in phase II trials $[72,73]$ tremelimumab, a human antibody against CTLA4, failed to demonstrate a benefit compared to placebo in a large randomized trial (mOS of 7.7 months vs. 7.3 months, respectively) [74].

The disappointing results of ICI monotherapy could be due both to the lack of biomarkers able to identify the proper candidate for immunotherapy, since PD-L1 expression seems not to be a reliable prognostic tool [75] and to the immunogenic characteristics of MPM. The limited mutation rate and the resultant low formation of antigens [76, 77] together with the potential upregulation of different inhibitory checkpoints, such as TIM-3 and LAG-3, resulting from anti-PD-1/PD-L1 and anti-CTLA4 drugs use [78], might explain the limited efficacy of ICIs.

\section{Double immune checkpoint inhibition}

In order to overcome these obstacles, the combination of two ICIs targeting different inhibitory checkpoints or the association of ICIs with chemotherapy might be a successful strategy.

The inhibition of PD-1 pathway, primarily involved in effector T-cell and NK-cells inhibition in peripheral tissue, and the blockade of CTLA4, which plays an important role in lymph nodes T-cell activation and in DCs suppression [79], demonstrated a synergistic effect in different types of cancer [80-82].

The phase II MAPS2 trial randomized patients to nivolumab or the combination of nivolumab and ipilimumab achieving the primary endpoint in both arms (DCR of $44.4 \%$ and $50 \%$ respectively). The survival analysis demonstrated a mOS 11.9 months in the nivolumab arm and 15.9 months for the combination arm [83]. On the basis of this trial, the FDA gave orphan drug designation to nivolumab or nivolumab plus ipilimumab in MPM patients after progression to first-line therapy.

The combination of tremelimumab and durvalumab was investigated in a phase II trial (NIBIT-MESO-1) as first and second-line treatment. This study enrolled patients with malignant pleural and peritoneal MPM and showed an ORR of $28 \%$ (reaching its primary endpoint), a DCR of $65 \%$, a mPFS of 8 months and a mOS of 16.6 months [84].

Similarly, the INITIATE trial investigated the activity of nivolumab with ipilimumab after first line treatment in MPM and peritoneal MPM patients and achieved the primary endpoint with DCR of 68\% [85]. 
The results of a prespecified interim analysis of a phase III randomized trial (CheckMate-743) [7] comparing the combination of nivolumab and ipilimumab vs. cisplatin/carboplatin and pemetrexed as first-line therapy in unresectable MPM were presented during the 2020 World Conference on Lung Cancer Virtual Presidential Symposium. A total of 605 patients were enrolled and, at a median follow-up of 29.7 months, the mOS was significantly longer for the nivolumab plus ipilimumab arm (18.1 months) than the chemotherapy arm (14.1 months). This study is the first randomized trial which demonstrated the superiority of ICIs combination over chemotherapy in first-line treatment for MPM patients, corroborating the rationale of dual immunotherapy strategy in this setting and leading to the recent approval by the FDA for this combination for first-line treatment in unresectable MPM adult patients.

\section{Chemotherapy + ICIs}

The combination of immunotherapy with chemotherapy is further a viable strategy as evidenced in malignancies including, for instance, breast cancer [86] and NSCLC [26, 27].

Beside the previously described mechanism for SCLC by which chemotherapy can broadly activate the immune system, an in vitro study investigated the effect of cisplatin, oxaliplatin and pemetrexed on three different MPM cells lines and highlighted a potential downregulation effect of cisplatin on immune checkpoints expression (PD-1, LAG-3, TIM-3) suggesting that this chemotherapeutic agent might be a promising partner for ICIs also in MPM [87].

The combination of durvalumab with cisplatin and pemetrexed chemotherapy as first-line therapy was investigated in a single arm phase II trial (DREAM study) which reported an ORR of $48 \%$ and a mPFS of 6.9 months [88].

The same combination was investigated in a recent phase II trial (PrE0505 study) which enrolled 55 patients with previously untreated unresectable MPM. This trial, presented at the virtual 2020 ASCO Annual Meeting, reached its primary endpoint, with a promising mOS of 20.4 months $(P=0.0014$, one-sided) [89].

The results of a phase II trial investigating pembrolizumab alone and in combination with cisplatin and pemetrexed in first-line setting are awaited (NCT02784171). The combination of ICI (nivolumab) with platinum-based chemotherapy is under investigation also in the adjuvant setting in a randomized controlled trial which is still recruiting (NCT04177953).

\section{Angiogenesis targeted agents + ICIs}

As previously discussed, tumors can evade immune response through VEGF-induced irregular vascularization and consequential T-cell infiltration hampering [90], suggesting that anti-VEGF agents could represent an ideal partner for ICIs to overcome immune resistance mechanisms.

Furthermore, given that VEGF exert a prominent role in MPM angiogenesis [91], anti-VEGF agents, either alone or in combination with chemotherapy, have been investigated in MPM patients, though with poor results [92-94]. The MAPS trial, a large phase III randomized study, investigated the combination of bevacizumab with cisplatin and pemetrexed, demonstrating a significantly enhanced mOS with the addition of the anti-VEGF agent (18.8 vs. 16.1 months, $P=0.0167)$ [95]. This triplet combination regimen can be considered as first-line treatment for MPM but, even if it has been validated by some US and European guidelines, it is not currently approved by the FDA and the European Medicines Agency (EMA) as the MAPS study was not a pivotal trial.

Against this background, the BEAT-meso trial was designed in order to investigate the efficacy of atezolizumab combined with bevacizumab and chemotherapy. In this phase III trial patients with unresectable MPM will be randomly assigned to treatment 1 group (4-6 cycles of carboplatin plus bevacizumab) or treatment 2 group (4-6 cycles of carboplatin plus bevacizumab plus atezolizumab). 
This multicentre trial is still recruiting, and its results are awaited as they could further revolutionize the treatment of this orphan disease.

\section{TETs}

TETs are a heterogeneous group of thoracic cancers, with an annual incidence of about 1.3 to 3.2 per million [96]. The World Health Organization classification stratified TETs into A, AB, B1, B2, and B3 thymomas and TC, taking into account characteristics of malignant epithelial cells and the percentage of non-neoplastic lymphocytes [97, 98]. TC represent about $10-12 \%$ of TETs and show a more aggressive clinical behavior and worse overall prognosis compared to thymomas [99]. If complete resection is possible due to early stage, surgery represents the first treatment choice. Locally advanced TETs require a multimodality approach characterized by chemotherapy and radiotherapy in addition to surgery. First-line platinum-based chemotherapy should be considered for advanced non-resectable or metastatic TETs [96]. Treatment options for relapsed or refractory disease after first-line are limited. Imatinib, a KIT TKI, may represent a therapy option for patients with TC who have progressed after first-line chemotherapy, in case of detection of $c$-KIT gene activating mutations $[100,101]$. Sunitinib, an anti-angiogenic multikinase inhibitor, could be used with the same indication, regardless from KIT status [102]. The thymus detains a key role in the development of immune tolerance and, through complex steps, leads to the development of central T-cell tolerance, necessary to avoid the onset of autoimmune diseases [103]. In the thymic medulla, the autoimmune regulator (AIRE) gene and the transcription factor FEZ family zinc finger 2 (FEZF2) promote the expression of tissue-specific antigens (TSAs) in order to develop T-cells. Those cells which react against TSAs undergo apoptosis [104, 105]. About $30 \%$ of thymoma patients develop autoimmune disorders, such as myasthenia gravis (the most frequent), pure red cell aplasia, hypogammaglobulinemia, systemic lupus erythematosus and pemphigus [106, 107]. This is due to the downregulation of AIRE and FEZF2, the overthrow of the normal thymic histological architecture, the deficient expression of MHC class II molecules by thymoma cells. These mechanisms lead to the failure of central immune tolerance and susceptibility to auto-immunity [108, 109].

Results obtained from several clinical trials with ICIs as monotherapy are encouraging for TETs showing promising outcome with a response rate (RR) of approximately $20 \%$ in pre-treated patient populations [110-113].

Giaccone et al. [110], assessed the activity of pembrolizumab in patients with advanced TC who had progressed after at least one line of chemotherapy, in a single-arm phase 2 study. The results were promising, in fact $22.5 \%$ of patients achieved a response; one (3\%) patient showed a complete response, eight (20\%) patients showed partial responses, and 21 (53\%) patients stable disease. Six (15\%) patients developed severe autoimmune toxicity, including two (5\%) patients with myocarditis. No deaths due to toxicity were observed.

Another phase II study evaluated the efficacy and safety of pembrolizumab in a cohort of 26 patients affected by TC and seven patients affected by thymoma, progressed after at least one line of platinum-based chemotherapy. Of seven thymomas, two (28.6\%) obtained partial response, and five (71.6\%) obtained stable disease. Of $26 \mathrm{TC}$, five (19.2\%) obtained partial response and 14 (53.8\%) stable disease. Taking into account immune-related adverse events (irAEs), five (71.4\%) of seven patients with thymoma and four (15.4\%) of 26 patients with TC reported grade $\geq 3$ irAEs, including myocarditis (three; 9.1\%) [111].

As shown by these trials, due to the physiological function of the thymus and the fact that TETs, mainly thymomas, are associated with defective immune tolerance, the use of immunotherapy could expose TETs patients to an increased risk for developing irAEs compared with patients with other malignancies. The incidence of irAEs is high in thymoma patients but also those with TC detain a greater risk of developing severe irAEs. In fact, about $15 \%$ of patients, compared to $6 \%$ of patients affected by other cancers, develop grade $\geq 3$ irAE, including potentially fatal myocarditis [111]. For this reason, an immune baseline checkup and a close monitoring of autoimmunity should be performed in TETs patients treated with ICIs, and their inclusion in clinical trials should be preferred. The detection of high tumor cells PD-L1 expression represents a strong rationale for using ICIs for treatment of TETs [114]. On the other hand, TMB, an emerging potential biomarker for ICIs efficacy, is very low in TETs $[115,116]$. In a wide cohort of 100 thymomas and 
69 TCs tissue expression of PD-L1, IDO and FOXP3 was analyzed and higher PD-L1 staining was detected in $36 \%$ of cases of both thymomas and TCs [114]. A work by Padda et al. [117], showed that TETs with higher PD-L1 expression detained a more aggressive histology (B3 thymomas and TCs) and worse prognosis. A meta-analysis about PD-L1 expression levels in TETs according to histological grade confirmed a significant higher PD-L1 positive rate in type B2/B3 thymoma and TC compared to the type A/AB/B1 thymoma group, suggesting that ICIs might be more effective for the former [118]. Interestingly, in a cohort of 43 patients affected by TCs, the PD-L1 tumor tissue staining increased after induction chemotherapy treatment [119]. This fact emphasizes that chemotherapy, but also targeted and epigenetic therapies [102, 120], holds immunomodulatory activities into the tumor microenvironment and that drug combinations with ICIs could be effective in a specific group of TETs patients. For example, belinostat, a pan-histone deacetylase inhibitor, detained immunomodulatory activity, leading to reduction in blood circulating Tregs and exhausted CD8 ${ }^{+}$ T-cell population of TETs patients included in the phase II trial [120], suggesting a potential synergy between epigenetic drugs and immunotherapy.

Considering the overexpression of VEGFA and VEGFR-1 and -2 [121] and the frequent PD-L1 expression both in TETs [122], anti-VEGF agents and ICIs represent an alternative combination strategy.

In a cohort of TETs patients treated with sunitinib (a multiple receptor TKI active against VEGFR-1, -2 and -3 , PDGFR- $\alpha$, PDGFR- $\beta$ and fibroblast growth factor receptor 1 ), higher expression of ICIs (i.e. CTLA4 and PD-1) was seen in circulating lymphocytes after sunitinib administration [102], suggesting its immunomodulatory effect and a potential synergism with ICIs. That represented the rationale for phase II trials that are currently evaluating pembrolizumab plus sunitinib and axitinib plus avelumab, respectively, for treatment of patients with TC. Another phase I/II trial is ongoing to assess the safety, tolerability and efficacy of nivolumab and vorolanib in combination in patients with thoracic tumors including TC. Vorolanib is a new selective VEGFR/PDGFR TKI designed to minimize side effects compared to other anti-angiogenic multikinase inhibitors $[123,124]$.

Finally, a phase II trial is available for TC patients to evaluate the efficacy of epacadost and pembrolizumab. Epacadostat is a potent indoleamine 2,3-dioxygenase 1 (ID01) enzyme inhibitor [125]. ID01 plays a central role in immune regulation through the catabolism of tryptophan to kynurenine [126]. Kynurenine and others tryptophan metabolites induce suppression of effector $\mathrm{CD}^{+} \mathrm{T}$ cells function, increase activity of Tregs, inhibit NK-cells and promote expansion of DCs and MDSCs. Therefore, cancers that express high levels of ID01 may elude immunosurveillance. In TETs, high expression of PD-L1 and IDO was observed in higher-grade forms of tumor histology [114] and for these reasons is reasonable to hypothesize a synergistic effect between epadacostat and pembrolizumab in patients affected by TC.

In conclusion, immunotherapy seems to be a promising therapeutic weapon in TETs, especially for TC. It is important to bear in mind that targeted therapies $[127,128]$ can also induce severe adverse effects and, due to their immune-modulation activity, potentially exacerbate irAEs linked to ICIs.

In order to better monitor TETs patients receiving immunotherapy, it would be appropriate to include patients in clinical trials and develop an appropriate and accurate monitoring plan that would allow early recognition of irAEs.

Discovering predictive factors, able to discriminate at baseline, before therapy start, patients more likely to develop potentially severe irAEs, would ultimately guide clinicians to the best therapeutic choice.

\section{Conclusions}

Since the introduction of PD-L1 and PD-1 inhibitors into the field of NSCLC, several clinical trials attempted to readapt these drugs in other thoracic malignancies. Although a modest single-agent activity has been seen in SCLC, MPM, and TETs, the benefits of ICI monotherapy have remained below expectations. The subtle but substantial differences between these tumor types and within the same tumor type are most probably responsible for delaying the strong affirmation of ICI monotherapy in this setting. Last evidences showed that the molecular profile and the prognosis of these rarer thoracic malignancies might be better explained by a continuous model rather than by a canonical categorical subdivision $[129,130]$. Similar to NSCLC, in which 
most of oncogene-driven tumors are highly resistant to ICIs, it is then improbable that all patients with SCLC, MPM, TETs will benefit from the same ICI strategy. In addition, it is now clear that the interaction between the tumor and the immune counterparts is considerably more complex and cannot be explained by just one immune checkpoint receptor. Spatial [131,132], temporal, and therapeutic [133] factors can affect checkpoints (both PD-1, PD-L1 and PD-L2) expression in tumor microenvironment. Taking into account these factors in the context of robust international trials supported by a proper outcome selection and strong translational analyses will be mandatory in the next future. As first approvals of anti-PD-1/PD-L1 in SCLC and MPM and also results from early-phase trials have recently shown, combining ICIs with chemotherapy, different ICIs (such as those targeting CTLA4) or even with targeted therapies, might represent a valid solution to overcome resistance and to broaden the population of thoracic cancer patients who may benefit from immunotherapy. However, further insight into potential biomarkers (both at tumor and at patient level) [12,130-133] as well as into the ideal timing/setting for immunotherapy is needed to get an upfront identification of patients who are likely to respond to these combination strategies and to avoid potentially harmful treatments. The reported toxicity profile of drug combinations draws clinical attention to both ongoing trials and real-world practice, with preexisting paraneoplastic disorders precluding the administration of ICIs combinations in some of these patients. Nevertheless, exploiting all advantages of ICIs in SCLC, MPM, and TETs is crucial, as very few other therapeutic options proved clinically relevant in these diseases.

\section{Abbreviations}

AIRE: autoimmune regulator

CIK: cytokine-induced killer

CTLA4: cytotoxic T-lymphocyte-associated protein 4

DC: dendritic cell

DCR: disease control rate

DDR: DNA damage response

ES-SCLC: extensive-small cell lung cancer

FEZF2: FEZ family zinc finger 2

ICD: immunogenic cell death

ICIs: immune checkpoint inhibitors

ID01: indoleamine 2,3-dioxygenase 1

irAEs: immune-related adverse events

ITT: intention-to-treat

MDSC: myeloid-derived suppressor cell

mOS: median overall survival

mPFS: median progression free survival

MPM: malignant pleural mesothelioma

NK-cell: natural killer cell

NSCLC: non-small cell lung cancer

ORR: objective response rate

OS: overall survival

PARP: poly (ADP-ribose) polymerase

PD-1: programmed cell death protein 1

PD-L1: programmed death-ligand 1

PD-L2: programmed death-ligand 2

PFS: progression-free survival 
SCLC: small cell lung cancer

TC: thymic carcinomas

TETs: thymic epithelial tumors

TMB: tumor mutation burden

Tregs: regulatory T cells

VEGF: vascular endothelial growth factor

\section{Declarations}

\section{Author contributions}

LC, FP, FM, AL, EL, FP wrote the manuscript and generated the figure and table. JA and RB contributed to the revisions of the manuscript. All authors approved the manuscript for publication.

\section{Conflicts of interest}

The authors declare that the research was conducted in the absence of any commercial or financial relationships that could be construed as a potential conflict of interest.

Relevant financial activities outside the submitted work: JA reports receiving commercial research grants from Amphera and Roche, holds ownership interest (including patents) in Amphera BV, and is a consultant/ advisory board member for Amphera, Boehringer Ingelheim, Bristol-Myers Squibb, Eli-Lilly, MSD, Takeda, Bayer, Astra Zeneca and Roche. RB is a consultant/advisory board member for Astra Zeneca, Boehringer Ingelheim, Novartis, MSD, Otsuka, Eli-Lilly, Roche. The other authors have no conflicts of interest to declare.

\section{Ethical approval}

Not applicable.

\section{Consent to participate}

Not applicable.

\section{Consent to publication}

Not applicable.

\section{Availability of data and materials}

Not applicable.

\section{Funding}

This research did not receive any specific grant from funding agencies in the public, commercial, or not-forprofit sectors.

\section{Copyright}

(c) The Author(s) 2021.

\section{References}

1. Reck M, Rodríguez-Abreu D, Robinson AG, Hui R, Csőszi T, Fülöp A, et al; KEYNOTE-024 Investigators. Pembrolizumab vs. chemotherapy for PD-L1-positive non-small-cell lung cancer. N Engl J Med. 2016;375:1823-33.

2. Gandhi L, Rodríguez-Abreu D, Gadgeel S, Esteban E, Felip E, De Angelis F, et al; KEYNOTE-189 Investigators. Pembrolizumab plus chemotherapy in metastatic non-small-cell lung cancer. $\mathrm{N}$ Engl J Med. 2018;378:2078-92. 
3. Cortellini A, Tiseo M, Banna GL, Cappuzzo F, Aerts JGJV, Barbieri F, et al. Clinicopathologic correlates of first-line pembrolizumab effectiveness in patients with advanced NSCLC and a PD-L1 expression of $\geq$ 50\%. Cancer Immunol Immunother. 2020;69:2209-21.

4. Cortellini A, Friedlaender A, Banna GL, Porzio G, Bersanelli M, Cappuzzo F, et al. Immune-related adverse events of pembrolizumab in a large real-world cohort of patients with NSCLC with a PD-L1 expression $\geq$ 50\% and their relationship with clinical outcomes. Clin Lung Cancer. 2020;21:498-508.e2.

5. Horn L, Mansfield AS, Szczęsna A, Havel L, Krzakowski M, Hochmair MJ, et al; IMpower133 Study Group. First-line atezolizumab plus chemotherapy in extensive-stage small-cell lung cancer. N Engl J Med. 2018;379:2220-9.

6. Paz-Ares L, Dvorkin M, Chen Y, Reinmuth N, Hotta K, Trukhin D, et al; CASPIAN investigators. Durvalumab plus platinum-etoposide $v s$. platinum-etoposide in first-line treatment of extensive-stage small-cell lung cancer (CASPIAN): a randomised, controlled, open-label, phase 3 trial. Lancet. 2019;394:1929-39.

7. Zalcman G, Peters S, Mansfield AS, Jahan TM, Popat S, Scherpereel A, et al. Checkmate 743: a phase 3, randomized, open-label trial of nivolumab (nivo) plus ipilimumab (ipi) vs. pemetrexed plus cisplatin or carboplatin as first-line therapy in unresectable pleural mesothelioma. J Clin Oncol. 2017;35 Suppl 15:TPS8581.

8. Paz-Ares L, Luft A, Vicente D, Tafreshi A, Gümüş M, Mazières J, et al; KEYNOTE-407 Investigators. Pembrolizumab plus chemotherapy for squamous non-small-cell lung cancer. $\mathrm{N}$ Engl $\mathrm{J}$ Med. 2018;379:2040-51.

9. Borghaei H, Paz-Ares L, Horn L, Spigel DR, Steins M, Ready NE, et al. Nivolumab vs. docetaxel in advanced nonsquamous non-small-cell lung cancer. N Engl J Med. 2015;373:1627-39.

10. Herbst RS, Baas P, Kim DW, Felip E, Pérez-Gracia JL, Han JY, et al. Pembrolizumab versus docetaxel for previously treated, PD-L1-positive, advanced non-small-cell lung cancer (KEYNOTE-010): a randomised controlled trial. Lancet. 2016;387:1540-50.

11. Hann CL, Scherpereel A, Hellyer JA, Wakelee HA. Role of immunotherapy in small cell lung cancer, thymic epithelial tumors, and mesothelioma. Am Soc Clin Oncol Educ Book. 2019;39:543-52.

12. Cantini L, Belderbos RA, Gooijer CJ, Dumoulin DW, Cornelissen R, Baart S, et al. Nivolumab in pre-treated malignant pleural mesothelioma: real-world data from the Dutch expanded access program. Transl Lung Cancer Res. 2020;9:1169-79.

13. Imbimbo M, Maury JM, Garassino M, Girard N; RARECAREnet Working Group. Mesothelioma and thymic tumors: treatment challenges in (outside) a network setting. Eur J Surg Oncol. 2019;45:75-80.

14. Pleasance ED, Stephens PJ, O'Meara S, McBride DJ, Meynert A, Jones D, et al. A small-cell lung cancer genome with complex signatures of tobacco exposure. Nature. 2010;463:184-90.

15. Chan TA, Yarchoan M, Jaffee E, Swanton C, Quezada SA, Stenzinger A, et al. Development of tumor mutation burden as an immunotherapy biomarker: utility for the oncology clinic. Ann Oncol. 2019;30:44-56.

16. Hellmann MD, Callahan MK, Awad MM, Calvo E, Ascierto PA, Atmaca A, et al. Tumor mutational burden and efficacy of nivolumab monotherapy and in combination with ipilimumab in small-cell lung cancer. Cancer Cell. 2018;33:853-61.e4.

17. George J, Lim JS, Jang SJ, Cun Y, Ozretić L, Kong G, et al. Comprehensive genomic profiles of small cell lung cancer. Nature. 2015;524:47-53.

18. Hellmann MD, Ott PA, Zugazagoitia J, Ready NE, Hann CL, De Braud FG, et al. Nivolumab (nivo) \pm ipilimumab (ipi) in advanced small-cell lung cancer (SCLC): first report of a randomized expansion cohort from CheckMate 032. J Clin Oncol. 2017;35 Suppl 15:8503.

19. Reck M, Vicente D, Ciuleanu T, Gettinger S, Peters S, Horn L, et al. Efficacy and safety of nivolumab (nivo) monotherapy versus chemotherapy (chemo) in recurrent small cell lung cancer (SCLC): results from CheckMate 331. Ann Oncol. 2018;29 Suppl 10:X43. 
20. Owonikoko TK, Kim HR, Govindan R, Ready N, Reck M, Peters S, et al. Nivolumab (nivo) plus ipilimumab (ipi), nivo, or placebo (pbo) as maintenance therapy in patients (pts) with extensive disease small cell lung cancer (ED-SCLC) after first-line (1L) platinum-based chemotherapy (chemo): results from the double-blind, rando. Ann Oncol. 2019;30 Suppl 2:ii77.

21. Chung HC, Piha-Paul SA, Lopez-Martin J, Schellens JHM, Kao S, Miller WH Jr, et al. Pembrolizumab after two or more lines of previous therapy in patients with recurrent or metastatic SCLC: results from the KEYNOTE-028 and KEYNOTE-158 studies. J Thorac Oncol. 2020;15:618-27.

22. Antonia SJ, López-Martin JA, Bendell J, Ott PA, Taylor M, Eder JP, et al. Nivolumab alone and nivolumab plus ipilimumab in recurrent small-cell lung cancer (CheckMate 032): a multicentre, open-label, phase 1/2 trial. Lancet Oncol. 2016;17:883-95.

23. Tian Y, Zhai X, Han A, Zhu H, Yu J. Potential immune escape mechanisms underlying the distinct clinical outcome of immune checkpoint blockades in small cell lung cancer. J Hematol Oncol. 2019;12:67.

24. Pavan A, Attili I, Pasello G, Guarneri V, Conte PF, Bonanno L. Immunotherapy in small-cell lung cancer: from molecular promises to clinical challenges. J Immunother Cancer. 2019;7:205.

25. Rossi A, Di Maio M, Chiodini P, Rudd RM, Okamoto H, Skarlos DV, et al. Carboplatin- or cisplatin-based chemotherapy in first-line treatment of small-cell lung cancer: the COCIS meta-analysis of individual patient data. J Clin Oncol. 2012;30:1692-8.

26. Hanna NH, Schneider BJ, Temin S, Baker S Jr, Brahmer J, Ellis PM, et al. Therapy for stage IV non-smallcell lung cancer without driver alterations: ASCO and $\mathrm{OH}$ (CCO) joint guideline update. J Clin Oncol. 2020;38:1608-32.

27. Socinski MA, Jotte RM, Cappuzzo F, Orlandi F, Stroyakovskiy D, Nogami N, et al. Atezolizumab for firstline treatment of metastatic nonsquamous NSCLC. N Engl J Med. 2018;378:2288-301.

28. Leonetti A, Wever B, Mazzaschi G, Assaraf YG, Rolfo C, Quaini F, et al. Molecular basis and rationale for combining immune checkpoint inhibitors with chemotherapy in non-small cell lung cancer. Drug Resist Updat. 2019;46:100644.

29. Zheng H, Zeltsman M, Zauderer MG, Eguchi T, Vaghjiani RG, Adusumilli PS. Chemotherapy-induced immunomodulation in non-small-cell lung cancer: a rationale for combination chemoimmunotherapy. Immunotherapy. 2017;9:913-27.

30. Rudin CM, Awad MM, Navarro A, Gottfried M, Peters S, Csőszi T, et al; KEYNOTE-604 Investigators. Pembrolizumab or placebo plus etoposide and platinum as first-line therapy for extensive-stage smallcell lung cancer: randomized, double-blind, phase III KEYNOTE-604 study. J Clin Oncol. 2020;38:2369-79.

31. Besse B, Menis J, Bironzo P, Gervais R, Greillier L, Monnet I, et al. REACTION: A phase II study of etoposide and cis/carboplatin with or without pembrolizumab in untreated extensive small cell lung cancer. Ann Oncol. 2020;31:S1211-2.

32. Reck M, Luft A, Szczesna A, Havel L, Kim SW, Akerley W, et al. Phase III randomized trial of ipilimumab plus etoposide and platinum versus placebo plus etoposide and platinum in extensive-stage small-cell lung cancer. J Clin Oncol. 2016;34:3740-8.

33. López-González A, Diz P, Gutierrez L, Almagro E, Palomo AG, Provencio M. The role of anthracyclines in small cell lung cancer. Ann Transl Med. 2013;1:5.

34. Sundstrøm S, Bremnes RM, Kaasa S, Aasebø U, Hatlevoll R, Dahle R, et al; Norwegian Lung Cancer Study Group. Cisplatin and etoposide regimen is superior to cyclophosphamide, epirubicin, and vincristine regimen in small-cell lung cancer: results from a randomized phase III trial with 5 years' follow-up. J Clin Oncol. 2002;20:4665-72.

35. Von Pawel J, Schiller JH, Shepherd FA, Fields SZ, Kleisbauer JP, Chrysson NG, et al. Topotecan versus cyclophosphamide, doxorubicin, and vincristine for the treatment of recurrent small-cell lung cancer. J Clin Oncol. 1999;17:658-67. 
36. Obeid M, Tesniere A, Ghiringhelli F, Fimia GM, Apetoh L, Perfettini JL, et al. Calreticulin exposure dictates the immunogenicity of cancer cell death. Nat Med. 2007; [Epub ahead of print].

37. Mankor JM, Zwierenga F, Dumoulin DW, Neefjes JJC, Aerts JGJV. A brief report on combination chemotherapy and anti-programmed death (ligand) 1 treatment in small-cell lung cancer: did we choose the optimal chemotherapy backbone? Eur J Cancer. 2020;137:40-4.

38. Forster M, Moreno V, Calvo E, Olmedo ME, Lopez-Criado MP, Lopez-Vilariño J, et al, et al. Overall survival with lurbinectedin plus doxorubicin in relapsed SCLC. Results from an expansion cohort of a phase Ib trial. J Thorac Oncol. 2018;13:S581.

39. Trigo Perez JM, Leary A, Besse B, Castellano DE, Ponce Aix S, Arrondeau J, et al. Efficacy and safety of lurbinectedin (PM1183) in small cell lung cancer (SCLC): results from a phase 2 study. J Clin Oncol. 2018;36 Suppl 15:8570.

40. Jiao S, Xia W, Yamaguchi H, Wei Y, Chen MK, Hsu JM, et al. PARP inhibitor upregulates PD-L1 expression and enhances cancer-associated immunosuppression. Clin Cancer Res. 2017;23:3711-20.

41. Sato H, Niimi A, Yasuhara T, Permata TBM, Hagiwara Y, Isono M, et al. DNA double-strand break repair pathway regulates PD-L1 expression in cancer cells. Nat Commun. 2017;8:1751.

42. Parkes EE, Walker SM, Taggart LE, McCabe N, Knight LA, Wilkinson R, et al. Activation of STINGdependent innate immune signaling by s-phase-specific DNA damage in breast cancer. J Natl Cancer Inst. 2017;109:djw199.

43. Sen T, Rodriguez BL, Chen L, Della Corte CM, Morikawa N, Fujimoto J, et al. Targeting DNA damage response promotes antitumor immunity through STING-mediated T-cell activation in small cell lung cancer. Cancer Discov. 2019;9:646-61.

44. Krebs M, Ross K, Kim S, De Jonge M, Barlesi F, Postel-Vinay S, et al. An open-label, multitumor phase ii basket study of olaparib and durvalumab (MEDIOLA): results in patients with relapsed SCLC. J Thorac Oncol. 2017;12:S2044-5.

45. Thomas A, Vilimas R, Trindade C, Erwin-Cohen R, Roper N, Xi L, et al. Durvalumab in combination with olaparib in patients with relapsed SCLC: results from a phase II study. J Thorac Oncol. 2019;14:1447-57.

46. Lok BH, Gardner EE, Schneeberger VE, Ni A, Desmeules P, Rekhtman N, et al. PARP inhibitor activity correlates with SLFN11 expression and demonstrates synergy with temozolomide in small cell lung cancer. Clin Cancer Res. 2017;23:523-35.

47. Stewart CA, Tong P, Cardnell RJ, Sen T, Li L, Gay CM, et al. Dynamic variations in epithelial-to-mesenchymal transition (EMT), ATM, and SLFN11 govern response to PARP inhibitors and cisplatin in small cell lung cancer. Oncotarget. 2017;8:28575-87.

48. Huang Y, Chen X, Dikov MM, Novitskiy SV, Mosse CA, Yang L, et al. Distinct roles of VEGFR-1 and VEGFR-2 in the aberrant hematopoiesis associated with elevated levels of VEGF. Blood. 2007;110:624-31.

49. Wu NZ, Klitzman B, Dodge R, Dewhirst MW. Diminished leukocyte-endothelium interaction in tumor microvessels. Cancer Res. 1992;52:4265-8.

50. Gabrilovich DI, Chen HL, Girgis KR, Cunningham HT, Meny GM, Nadaf S, et al. Production of vascular endothelial growth factor by human tumors inhibits the functional maturation of dendritic cells. Nat Med. 1996;2:1096-103.

51. Huang Y, Yuan J, Righi E, Kamoun WS, Ancukiewicz M, Nezivar J, et al. Vascular normalizing doses of antiangiogenic treatment reprogram the immunosuppressive tumor microenvironment and enhance immunotherapy. Proc Natl Acad Sci U S A. 2012;109:17561-6.

52. Tanno S, Ohsaki Y, Nakanishi K, Toyoshima E, Kikuchi K. Human small cell lung cancer cells express functional VEGF receptors, VEGFR-2 and VEGFR-3. Lung Cancer. 2004;46:11-9.

53. Schultheis AM, Scheel AH, Ozretić L, George J, Thomas RK, Hagemann T, et al. PD-L1 expression in small cell neuroendocrine carcinomas. Eur J Cancer. 2015;51:421-6. 
54. Meder L, Schuldt P, Thelen M, Schmitt A, Dietlein F, Klein S, et al. Combined VEGF and PD-L1 blockade displays synergistic treatment effects in an autochthonous mouse model of small cell lung cancer. Cancer Res. 2018;78:4270-81.

55. Nan X, Xie C, Yu X, Liu J. EGFR TKI as first-line treatment for patients with advanced EGFR mutationpositive non-small-cell lung cancer. Oncotarget. 2017;8:75712-26.

56. Yakes FM, Chen J, Tan J, Yamaguchi K, Shi Y, Yu P, et al. Cabozantinib (XL184), a novel MET and VEGFR2 inhibitor, simultaneously suppresses metastasis, angiogenesis, and tumor growth. Mol Cancer Ther. 2011;10:2298-308.

57. Yu L, Lai Q, Gou L, Feng J, Yang J. Opportunities and obstacles of targeted therapy and immunotherapy in small cell lung cancer. J Drug Target. 2021;29:1-11.

58. Andujar P, Lacourt A, Brochard P, Pairon JC, Jaurand MC, Jean D. Five years update on relationships between malignant pleural mesothelioma and exposure to asbestos and other elongated mineral particles. J Toxicol Environ Health Part B Crit Rev. 2016;19:151-72.

59. Vogelzang NJ, Rusthoven JJ, Symanowski J, Denham C, Kaukel E, Ruffie P, et al. Phase III study of pemetrexed in combination with cisplatin versus cisplatin alone in patients with malignant pleural mesothelioma. J Clin Oncol. 2003;21:2636-44.

60. Baas P, Fennell D, Kerr KM, Vn Schil PE, Haas RL, Peters S. Malignant pleural mesothelioma: ESMO Clinical Practice Guidelines for diagnosis, treatment and follow-up. Ann Oncol. 2015;26 Suppl 5:v31-9.

61. Astoul P, Roca E, Galateau-Salle F, Scherpereel A. Malignant pleural mesothelioma: from the bench to the bedside. Respiration. 2012;83:481-93.

62. Kumar T, Patel N, Talwar A. Spontaneous regression of thoracic malignancies. Respir Med. 2010;104:1543-50.

63. Yamada N, Oizumi S, Kikuchi E, Shinagawa N, Konishi-Sakakibara J, Ishimine A, et al. CD8 ${ }^{+}$tumorinfiltrating lymphocytes predict favorable prognosis in malignant pleural mesothelioma after resection. Cancer Immunol Immunother. 2010;59:1543-9.

64. Cornelissen R, Lievense LA, Maat AP, Hendriks RW, Hoogsteden HC, Bogers AJ, et al. Ratio of intratumoral macrophage phenotypes is a prognostic factor in epithelioid malignant pleural mesothelioma. PLoS One. 2014;9:e106742.

65. Mansfield AS, Roden AC, Peikert T, Sheinin YM, Harrington SM, Krco CJ, et al. B7-H1 expression in malignant pleural mesothelioma is associated with sarcomatoid histology and poor prognosis. J Thorac Oncol. 2014;9:1036-40.

66. Desai A, Karrison T, Rose B, Tan Y, Hill B, Pemberton E, et al. OA08.03 Phase II trial of pembrolizumab (NCT02399371) in previously-treated malignant mesothelioma (MM): final analysis. J Thorac Oncol. 2018;13:S339.

67. Quispel-Janssen J, van der Noort V, de Vries JF, Zimmerman M, Lalezari F, Thunnissen E, et al. Programmed death 1 blockade with nivolumab in patients with recurrent malignant pleural mesothelioma. J Thorac Oncol. 2018;13:1569-76.

68. Okada M, Kijima T, Aoe K, Kato T, Fujimoto N, Nakagawa K, et al. Clinical efficacy and safety of nivolumab: results of a multicenter, open-label, single-arm, Japanese phase 2 study in malignant pleural mesothelioma (MERIT). Clin Cancer Res. 2019;25:5482-92.

69. Hassan R, Thomas A, Nemunaitis JJ, Patel MR, Bennouna J, Chen FL, et al. Efficacy and safety of avelumab treatment in patients with advanced unresectable mesothelioma: phase $1 \mathrm{~b}$ results from the JAVELIN solid tumor trial. JAMA Oncol. 2019;5:351-7.

70. Popat S, Curioni-Fontecedro A, Polydoropoulou V, Shah R, O’Brien M, Pope A, et al. LBA91_PRA multicentre randomized phase III trial comparing pembrolizumab (P) vs. single agent chemotherapy (CT) for advanced pre-treated malignant pleural mesothelioma (MPM): results from the European Thoracic Oncology Platform (ETOP 9-15) PROMISE-meso trial. Ann Oncol. 2019;30 Suppl 5:v931. 
71. Fennell DA, Kirkpatrick E, Cozens K, Nye M, Lester J, Hanna G, et al. CONFIRM: a double-blind, placebocontrolled phase III clinical trial investigating the effect of nivolumab in patients with relapsed mesothelioma: study protocol for a randomised controlled trial. Trials. 2018;19:233.

72. Calabrò L, Morra A, Fonsatti E, Cutaia O, Amato G, Giannarelli D, et al. Tremelimumab for patients with chemotherapy-resistant advanced malignant mesothelioma: an open-label, single-arm, phase 2 trial. Lancet Oncol. 2013;14:1104-11.

73. Calabrò L, Morra A, Fonsatti E, Cutaia O, Fazio C, Annesi D, et al. Efficacy and safety of an intensified schedule of tremelimumab for chemotherapy-resistant malignant mesothelioma: an open-label, singlearm, phase 2 study. Lancet Respir Med. 2015;3:301-9.

74. Maio M, Scherpereel A, Calabrò L, Aerts J, Perez SC, Bearz A, et al. Tremelimumab as second-line or third-line treatment in relapsed malignant mesothelioma (DETERMINE): a multicentre, international, randomised, double-blind, placebo-controlled phase 2b trial. Lancet Oncol. 2017;18:1261-73.

75. Alley EW, Lopez J, Santoro A, Morosky A, Saraf S, Piperdi B, et al. Clinical safety and activity of pembrolizumab in patients with malignant pleural mesothelioma (KEYNOTE-028): preliminary results from a non-randomised, open-label, phase $1 \mathrm{~b}$ trial. Lancet Oncol. 2017;18:623-30.

76. Cinausero M, Rihawi K, Cortiula F, Follador A, Fasola G, Ardizzoni A. Emerging therapies in malignant pleural mesothelioma. Crit Rev Oncol Hematol. 2019;144:102815.

77. Cantini L, Hassan R, Sterman DH, Aerts JGJV. Emerging treatments for malignant pleural mesothelioma: where are we heading? Front Oncol. 2020;10:343.

78. Dozier J, Zheng H, Adusumilli PS. Immunotherapy for malignant pleural mesothelioma: current status and future directions. Transl Lung Cancer Res. 2017;6:315-24.

79. Rotte A. Combination of CTLA-4 and PD-1 blockers for treatment of cancer. J Exp Clin Cancer Res. 2019;38:255.

80. Wolchok JD, Chiarion-Sileni V, Gonzalez R, Rutkowski P, Grob JJ, Cowey CL, et al. Overall survival with combined nivolumab and ipilimumab in advanced melanoma. N Engl J Med. 2017;377:1345-56.

81. Motzer RJ, Tannir NM, McDermott DF, Arén Frontera O, Melichar B, Choueiri TK, et al. Nivolumab plus ipilimumab vs. sunitinib in advanced renal-cell carcinoma. N Engl J Med. 2018;378:1277-90.

82. Overman MJ, Lonardi S, Wong KYM, Lenz HJ, Gelsomino F, Aglietta M, et al. Durable clinical benefit with nivolumab plus ipilimumab in DNA mismatch repair-deficient/microsatellite instability-high metastatic colorectal cancer. J Clin Oncol. 2018;36:773-79.

83. Zalcman G, Mazieres J, Greillier L, Brosseau S, Lantuejoul S, Do P, et al. Second/third-line nivolumab vs. nivo plus ipilimumab in malignant pleural mesothelioma: long-term results of IFCT-1501 MAPS2 phase IIR trial with a focus on hyperprogression (HPD). Ann Oncol. 2019;30 Suppl 5:V747.

84. Calabrò L, Morra A, Giannarelli D, Amato G, D'Incecco A, Covre A, et al. Tremelimumab combined with durvalumab in patients with mesothelioma (NIBIT-MESO-1): an open-label, non-randomised, phase 2 study. Lancet Respir Med. 2018;6:451-60.

85. Disselhorst MJ, Quispel-Janssen J, Lalezari F, Monkhorst K, de Vries JF, van der Noort V, et al. Ipilimumab and nivolumab in the treatment of recurrent malignant pleural mesothelioma (INITIATE): results of a prospective, single-arm, phase 2 trial. Lancet Respir Med. 2019;7:260-70.

86. Schmid P, Adams S, Rugo HS, Schneeweiss A, Barrios CH, Iwata H, et al. Atezolizumab and nab-paclitaxel in advanced triple-negative breast cancer. N Engl J Med. 2018;379:2108-21.

87. Marcq E, Audenaerde JRV, Waele J, Jacobs J, Loenhout JV, Cavents G, et al. Building a bridge between chemotherapy and immunotherapy in malignant pleural mesothelioma: investigating the effect of chemotherapy on immune checkpoint expression. Int J Mol Sci. 2019;20:4182.

88. Nowak A, Kok P, Lesterhuis W, Hughes B, Brown C, Kao S, et al. OA08.02 DREAM-A phase 2 trial of durvalumab with first line chemotherapy in mesothelioma: final result. J Thorac Oncol. 2018;13:S338-9. 
89. Forde PM, Sun Z, Anagnostou V, Kindler HL, Purcell WT, Goulart BHL, et al. Phase II multicenter study of anti-PD-L1, durvalumab, in combination with cisplatin and pemetrexed for the first-line treatment of unresectable malignant pleural mesothelioma (MPM)-a PrECOG LLC study. J Clin Oncol. 2020;38 Suppl 15:9003.

90. Kim JM, Chen DS. Immune escape to PD-L1/PD-1 blockade: seven steps to success (or failure). Ann Oncol. 2016;27:1492-504.

91. Ohta Y, Shridhar V, Bright RK, Kalemkerian GP, Du W, Carbone M, et al. VEGF and VEGF type C play an important role in angiogenesis and lymphangiogenesis in human malignant mesothelioma tumours. $\mathrm{Br}$ J Cancer. 1999;81:54-61.

92. Garland LL, Chansky K, Wozniak AJ, Tsao AS, Gadgeel SM, Verschraegen CF, et al. Phase II study of cediranib in patients with malignant pleural mesothelioma: SWOG S0509. J Thorac Oncol. 2011;6:1938-45.

93. Buikhuisen WA, Burgers JA, Vincent AD, Korse CM, van Klaveren RJ, Schramel FM, et al. Thalidomide versus active supportive care for maintenance in patients with malignant mesothelioma after firstline chemotherapy (NVALT 5): an open-label, multicentre, randomised phase 3 study. Lancet Oncol. 2013;14:543-51.

94. Buikhuisen WA, Scharpfenecker M, Griffioen AW, Korse CM, Van Tinteren H, Baas P. A randomized phase II study adding axitinib to pemetrexed-cisplatin in patients with malignant pleural mesothelioma: a single-center trial combining clinical and translational outcomes. J Thorac Oncol. 2016;11:758-68.

95. Zalcman G, Mazieres J, Margery J, Greillier L, Audigier-Valette C, Moro-Sibilot D, et al. Bevacizumab for newly diagnosed pleural mesothelioma in the Mesothelioma Avastin Cisplatin Pemetrexed Study (MAPS): a randomised, controlled, open-label, phase 3 trial. Lancet. 2016;387:1405-14.

96. Girard N, Ruffini E, Marx A, Faivre-Finn C, Peters S; ESMO Guidelines Committee. Thymic epithelial tumours: ESMO Clinical Practice Guidelines for diagnosis, treatment and follow-up. Ann Oncol. 2015;26 Suppl 5:v40-55.

97. Travis WD, Brambilla E, Burke AP, Marx A, Nicholson AG, editors. WHO classification of tumours of the lung, pleura, thymus and heart. 4th ed. Lyon: IARC Publications; 2015.

98. den Bakker MA, Roden AC, Marx A, Marino M. Histologic classification of thymoma: a practical guide for routine cases. J Thorac Oncol. 2014;9 Suppl 2:S125-30.

99. Liu HC, Hsu WH, Chen YJ, Chan YJ, Wu YC, Huang BS, et al. Primary thymic carcinoma. Ann Thorac Surg. 2002;73:1076-81.

100. Lamarca A, Moreno V, Feliu J. Thymoma and thymic carcinoma in the target therapies era. Cancer Treat Rev. 2013;39:413-20.

101. Ströbel P, Hartmann M, Jakob A, Mikesch K, Brink I, Dirnhofer S, et al. Thymic carcinoma with overexpression of mutated KIT and the response to imatinib. N Engl J Med. 2004;350:2625-6.

102. Thomas A, Rajan A, Berman A, Tomita Y, Brzezniak C, Lee MJ, et al. Sunitinib in patients with chemotherapyrefractory thymoma and thymic carcinoma: an open-label phase 2 trial. Lancet Oncol. 2015;16:177-86.

103. Cheng M, Anderson MS. Thymic tolerance as a key brake on autoimmunity. Nat Immunol. 2018;19:659-64.

104. Perniola R. Twenty years of AIRE. Frontiers in Immunology. Front Immunol. 2018;9:98.

105. Malchow S, Leventhal DS, Nishi S, Fischer BI, Shen L, Paner GP, et al. Aire-dependent thymic development of tumor-associated regulatory T cells. Science. 2013;339:1219-24.

106. Tseng YC, Hsieh CC, Huang HY, Huang CS, Hsu WH, Huang BS, et al. Is thymectomy necessary in nonmyasthenic patients with early thymoma? J Thorac Oncol. 2013;8:952-8.

107. Marx A, Willcox N, Leite MI, Chuang WY, Schalke B, Nix W, et al. Thymoma and paraneoplastic myasthenia gravis. Autoimmunity. 2010;43:413-27.

108. Machens A, Löliger C, Pichlmeier U, Emskötter T, Busch C, Izbicki JR. Correlation of thymic pathology with HLA in myasthenia gravis. Clin Immunol. 1999;91:296-301. 
109. Radovich M, Pickering CR, Felau I, Ha G, Zhang H, Jo H, et al. The integrated genomic landscape of thymic epithelial tumors. Cancer Cell. 2018;33:244-58.e10.

110. Giaccone G, Kim C, Thompson J, McGuire C, Kallakury B, Chahine JJ, et al. Pembrolizumab in patients with thymic carcinoma: a single-arm, single-centre, phase 2 study. Lancet Oncol. 2018;19:347-55.

111. Cho J, Kim HS, Ku BM, Choi YL, Cristescu R, Han J, et al. Pembrolizumab for patients with refractory or relapsed thymic epithelial tumor: an open-label phase II trial. J Clin Oncol. 2019;37:2162-70.

112. Seto T, Katsuya Y, Horinouchi H, Umemura S, Hosomi Y, Satouchi M, et al. Primary result of an investigatorinitiated phase II trial of nivolumab for unresectable or recurrent thymic carcinoma: PRIMER study (NCCH1505). J Thorac Oncol. 2018;13:S61-2.

113. Rajan A, Heery CR, Thomas A, Mammen AL, Perry S, O'Sullivan Coyne G, et al. Efficacy and tolerability of anti-programmed death-ligand 1 (PD-L1) antibody (Avelumab) treatment in advanced thymoma. J Immunother Cancer. 2019;7:269.

114. Wei YF, Chu CY, Chang CC, Lin SH, Su WC, Tseng YL, et al. Different pattern of PD-L1, IDO, and FOXP3 Tregs expression with survival in thymoma and thymic carcinoma. Lung Cancer. 2018;125:35-42.

115. Ross JS, Gay LM, Wang K, Vergilio JA, Suh J, Ramkissoon S, et al. Comprehensive genomic profiles of metastatic and relapsed salivary gland carcinomas are associated with tumor type and reveal new routes to targeted therapies. Ann Oncol. 2017;28:2539-46.

116. Rajan A, Girard N, Marx A. State of the art of genetic alterations in thymic epithelial tumors. J Thorac Oncol. 2014;9 Suppl 2:S131-6.

117. Padda SK, Riess JW, Schwartz EJ, Tian L, Kohrt HE, Neal JW, et al. Diffuse high intensity PD-L1 staining in thymic epithelial tumors. J Thorac Oncol. 2015;10:500-8.

118. Chen HF, Wu LX, Li XF, Zhu YC, Pan WW, Wang WX, et al. PD-L1 expression level in different thymoma stages and thymic carcinoma: a meta-analysis. Tumori. 2020;106:306-11.

119. Funaki S, Shintani Y, Fukui E, Yamamoto Y, Kanzaki R, Ose N, et al. The prognostic impact of programmed cell death 1 and its ligand and the correlation with epithelial-mesenchymal transition in thymic carcinoma. Cancer Med. 2019;8:216-26.

120. Giaccone G, Rajan A, Berman A, Kelly RJ, Szabo E, Lopez-Chavez A, et al. Phase II study of belinostat in patients with recurrent or refractory advanced thymic epithelial tumors. J Clin Oncol. 2011;29:2052-9.

121. Cimpean AM, Raica M, Encica S, Cornea R, Bocan V. Immunohistochemical expression of vascular endothelial growth factor A (VEGF), and its receptors (VEGFR1, 2) in normal and pathologic conditions of the human thymus. Ann Anat. 2008;190:238-45.

122. Arbour KC, Naidoo J, Steele KE, Ni A, Moreira AL, Rekhtman N, et al. Expression of PD-L1 and other immunotherapeutic targets in thymic epithelial tumors. PLoS One. 2017;12:e0182665.

123. Sheng X, Yan X, Tang B, Chi Z, Cui C, Si L, et al. A phase I clinical trial of CM082 (X-82) in combination with everolimus for treatment of metastatic renal cell carcinoma. J Clin Oncol. 2017;35 Suppl 15:4575.

124. Yan X, Sheng X, Tang B, Chi Z, Cui C, Si L, et al. Anti-VEGFR, PDGFR, and CSF1R tyrosine kinase inhibitor CM082 (X-82) in combination with everolimus for treatment of metastatic renal cell carcinoma: a phase 1 clinical trial. Lancet Oncol. 2017;18:S8.

125. Yue EW, Sparks R, Polam P, Modi D, Douty B, Wayland B, et al. INCB24360 (Epacadostat), a highly potent and selective indoleamine-2,3-dioxygenase 1 (IDO1) inhibitor for immuno-oncology. ACS Med Chem Lett. 2017;8:486-91.

126. Brochez L, Chevolet I, Kruse V. The rationale of indoleamine 2,3-dioxygenase inhibition for cancer therapy. European Journal of Cancer. Eur J Cancer. 2017;76:167-82.

127. Ravaud A, Urva SR, Grosch K, Cheung WK, Anak O, Sellami DB. Relationship between everolimus exposure and safety and efficacy: meta-analysis of clinical trials in oncology. Eur J Cancer. 2014;50:486-95. 
128. Rajan A, Carter CA, Berman A, Cao L, Kelly RJ, Thomas A, et al. Cixutumumab for patients with recurrent or refractory advanced thymic epithelial tumours: a multicentre, open-label, phase 2 trial. Lancet Oncol. 2014;15:191-200.

129. Alcala N, Mangiante L, Le-Stang N, Gustafson CE, Boyault S, Damiola F, et al. Redefining malignant pleural mesothelioma types as a continuum uncovers immune-vascular interactions. EBioMedicine. 2019;48:191-202.

130. Centonze G, Biganzoli D, Prinzi N, Pusceddu S, Mangogna A, Tamborini E, et al. Beyond traditional morphological characterization of lung neuroendocrine neoplasms: in silico study of next-generation sequencing mutations analysis across the four world health organization defined groups. Cancers (Basel). 2020;12:2753.

131. Ilie M, Long-Mira E, Bence C, Butori C, Lassalle S, Bouhlel L, et al. Comparative study of the PD-L1 status between surgically resected specimens and matched biopsies of NSCLC patients reveal major discordances: a potential issue for anti-PD-L1 therapeutic strategies. Ann Oncol. 2016;27:147-53.

132. McLaughlin J, Han G, Schalper KA, Carvajal-Hausdorf D, Pelekanou V, Rehman J, et al. Quantitative assessment of the heterogeneity of PD-L1 expression in non-small-cell lung cancer. JAMA Oncol. 2016;2:46-54.

133. Sheng J, Fang W, Yu J, Chen N, Zhan J, Ma Y, et al. Expression of programmed death ligand-1 on tumor cells varies pre and post chemotherapy in non-small cell lung cancer. Sci Rep. 2016;6:20090. 